\title{
Thoracic aortic stent grafting: Improving results with newer generation investigational devices
}

Jehangir J. Appoo, MD, William G. Moser, RN, Ronald M. Fairman, MD, Katherine F. Cornelius, RN, A. Pochettino, MD, Edward Y. Woo, MD, Jibby E. Kurichi, MPH, Jefferey P. Carpenter, MD, and Joseph E. Bavaria, MD

From the Division of Cardiothoracic Surgery and Division of Vascular Surgery, Hospital of the University of Pennsylvania, Philadelphia, Pa.

Read at the Thirty-first Annual Meeting of The Western Thoracic Surgical Association, Victoria, BC, Canada, June 22-25, 2005.

Received for publication June 27, 2005; revisions received Nov 28, 2005; accepted for publication Dec 22, 2005.

Address for reprints: Joseph E. Bavaria, MD, Brooke Roberts Professor of Surgery, Division of Cardiothoracic Surgery, 4 Silverstein, Hospital of University of Pennsylvania, 3400 Spruce Street, Philadelphia, PA 19104 (E-mail: joseph.bavaria@uphs. upenn.edu).

J Thorac Cardiovasc Surg 2006;131:1087-94 $0022-5223 / \$ 32.00$

Copyright $\odot 2006$ by The American Association for Thoracic Surgery

doi:10.1016/j.jtcvs.2005.12.058
Objective: Six years ago an endovascular program for repair of descending thoracic aneurysms was established at the University of Pennsylvania. We report on the hypothesis that results are improving with new stent design iterations and describe our experience and lessons learned.

Methods: From April 1999 to March 2005, 99 patients with descending thoracic aneurysms underwent repair with a first or second-generation commercially produced endograft; 24 patients had an early-generation device, and 75 patients had a late-generation device. Each patient was enrolled as part of 3 distinct Phase I or Phase II Food and Drug Administration-approved clinical trials in accordance with strict inclusion and exclusion criteria.

Results: Mean age was 73.1 years. Symptomatic aneurysms accounted for $42 \%$ of the cohort. Mean aneurysm size was $63.7 \mathrm{~mm}$ (range: 30-105 mm). Twenty percent of the patients underwent a subclavian carotid transposition or bypass preoperatively to obtain an adequate proximal landing zone. No procedures had to be aborted. In-hospital or 30-day mortality was $5.0 \%$. The incidence of permanent spinal ischemia was $2 \%$. Perioperative vascular complications requiring interposition graft, stent repair, or patch angioplasty occurred in $27 \%$ and seemed to be less frequent in the late-generation cohort than the early-generation cohort $(22.7 \%$ vs $41.7 \%$, respectively, $P=.069)$. At the 30-day follow-up, 23 endoleaks were detected in 22 patients $(14.7 \%$ in late-generation cohort vs $45.8 \%$ in early-generation cohort, $P=$ .001). During the follow-up period, 3 new endoleaks were detected, 3 patients died of aortic rupture, and 10 patients underwent aneurysm-related reintervention. Kaplan-Meier estimated 1, 3, and 5-year survival was $84.5 \%, 70.5 \%$, and $52.4 \%$, respectively. Freedom from aneurysm-related event, defined as freedom from endoleak, aortic rupture, dissection, or any reintervention on the aorta, was $73 \%, 69 \%$, and $64 \%$ at 1,3 , and 5 years, respectively.

Conclusion: Thoracic aortic stent grafting is a safe procedure in selected patients with the added benefit of a low incidence of paraplegia. However, there is an incidence of late complications and reinterventions. This risk requires further quantification and must be balanced against the benefits of a minimally invasive approach with low perioperative morbidity and mortality. Results are improving as technology evolves and our level of experience increases. Radiologic follow-up is mandatory.

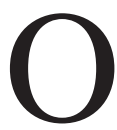
pen repair of a descending thoracic aneurysm (DTA) is still associated with significant morbidity and mortality. ${ }^{1,2}$ Recent refinements in open technique, including extracorporeal circulation for peripheral organ preservation and multiple techniques for spinal cord protection, may improve results but are not applied universally. The highly invasive nature of this procedure necessitates a prolonged recovery period. In addition, high-risk patients who were previously denied surgical repair because of comorbidities, including but not limited to pul- 

Abbreviations and Acronyms
$\mathrm{CT}=$ computed tomography
DTA $=$ descending thoracic aneurysm
FDA $=$ Food and Drug Administration

monary, cardiac, and renal status, may become surgical candidates if a less-invasive endovascular option was possible. For these reasons, endovascular repair is preferable.

The objectives of this article are to report on the hypothesis that results are improving with new stent design iterations and to describe our experience over a 6-year period.

\section{Methods}

This report analyzes 99 of 128 endografts for DTA from April 1, 1999, to March 31, 2005, performed at the University of Pennsylvania. Included were all patients entered in clinical trials approved by the Food and Drug Administration (FDA). Excluded were homemade devices, compassionate use indications, and off-label (out of trial) uses of endografts. Data were prospectively entered into a clinical database. All protocols and procedures were approved by the FDA, and consent was obtained from the institutional review board. All patients gave informed consent before inclusion in this study.

Early-generation devices were inserted in high-risk patients enrolled in a Phase I study: Investigation of the TALENT Endoluminal Spring Stent Graft System for the Treatment of Thoracic Aortic Aneurysms in High-Risk Patients.

Late-generation devices were inserted in patients who were operative candidates enrolled in 1 of the following Phase 2 trials:

1. Evaluation of the Medtronic AVE Talent Thoracic Stent Graft System for the Treatment of Thoracic Aortic Aneurysms: The VALOR trial

2. A Clinical Study Comparing Use of the Thoracic Excluder Endoprosthesis to Open Surgical Repair in the Primary Treatment of Descending Thoracic Aneurysms (TAG 99-01)

3. Treatment IDE for Use of the GORE TAG Thoracic Endoprosthesis in Subjects with Descending Thoracic Aortic Aneurysms Requiring Surgical Repair

A surgical team consisting of a cardiac surgeon and vascular surgeon performed all operations. Operations were performed under general anesthesia in the cardiac surgery or vascular surgery operating theatre. Recently, a fully equipped endovascular suite was built in the main operating room. All patients recovered postoperatively in the cardiac surgical intensive care unit.

Use of cerebrospinal fluid drains and somatosensory evoked potential monitoring was not standardized in this population but evolved with increasing experience. In our current management paradigm, preoperative lumbar drains and intraoperative somatosensory evoked potential monitoring are used in all patients who are considered high risk for spinal ischemia. These include the following:

- previous abdominal aortic aneurysm repair

- previous descending thoracic aortic surgery

- planned complete aortic coverage from left subclavian to celiac axis (type $\mathrm{C}$, Figure 1)
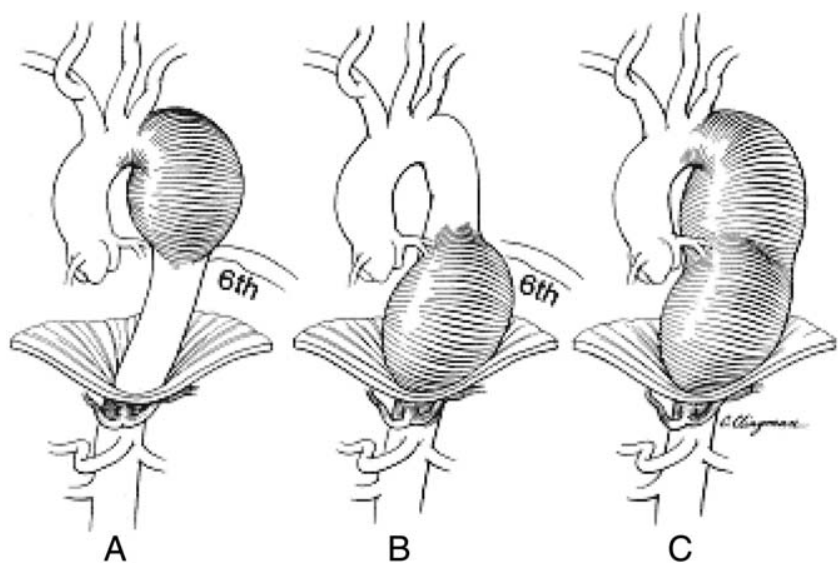

Figure 1. Classification of descending thoracic aneurysms (DTAs). Descending thoracic aortic aneurysms are classified as type $A$, left subclavian artery to T6 (A); type B, midthoracic aorta to celiac axis (B); or type C, left subclavian to celiac axis (C).

All patients who had planned left subclavian artery coverage underwent preoperative elective left carotid-subclavian bypass. All patients with DTAs were considered for both endovascular repair and open repair by the same surgical team. If the aneurysm profile did not fit the inclusion/exclusion criteria of the respective trials or patients did not consent to endovascular repair, an open repair was conducted with the use of left-sided heart bypass.

\section{Preoperative Imaging}

All potential candidates underwent preoperative aortography or high-definition computed tomography (CT) scanning. CT scans were then digitally reformatted into 3 dimensional $1-\mathrm{mm}$ cuts using MMS technology (Medical Metrx Solutions, West Lebanon, $\mathrm{NH}$ ), providing a patient-specific model that assists in understanding the relationships between structures and helps in accurately designing a stent graft system. Cerebrovascular assessment was based on angiographically determined vertebral artery patency. No attempt was made at preoperatively imaging intercostal arteries that may supply the spinal cord.

\section{Statistical Analysis}

All statistical tests were 2-tailed. Student $t$ tests, chi-square tests, and Fisher exact tests were conducted when appropriate to assess differences between groups. Overall survival and freedom from aneurysm-related event curves were calculated by the KaplanMeier method. Calculations were performed using SPSS (SPSS for Windows, Version 12.0, SPSS Inc. Chicago, Ill).

\section{Results}

\section{Patient Characteristics}

A total of 99 patients satisfied all inclusion and exclusion criteria between April 1, 1999, and March 31, 2005, and were enrolled to receive an endograft; 24 patients received an early-generation endograft, and 75 patients received a lategeneration endograft. Patient demographics are outlined in 
TABLE 1. Patient demographics

\begin{tabular}{|c|c|c|c|c|}
\hline & $\begin{array}{l}\text { Early generation } \\
\qquad N=24\end{array}$ & $\begin{array}{l}\text { Late generation } \\
\qquad \mathbf{N}=75\end{array}$ & $\begin{array}{c}\text { Overall University of } \\
\text { Pennsylvania experience } \\
\qquad \mathrm{N}=99\end{array}$ & $\begin{array}{c}P \text { value } \\
\text { (early vs late generation) }\end{array}$ \\
\hline Mean age (y) & 75.62 & 72 & 73.1 & .201 \\
\hline \multicolumn{5}{|l|}{ Sex: } \\
\hline Male & $16(66.7 \%)$ & $41(54.7 \%)$ & 57 & .301 \\
\hline Female & $8(33.3 \%)$ & $34(45.3 \%)$ & 42 & .301 \\
\hline CAD & $15(62.5 \%)$ & $31(41.3 \%)$ & 46 & .07 \\
\hline COPD & $19(79.2 \%)$ & $35(46.7 \%)$ & 54 & .005 \\
\hline Smoking & $21(87.5 \%)$ & $58(77.3 \%)$ & 79 & .28 \\
\hline HTN & $19(79.2 \%)$ & $50(66.7 \%)$ & 69 & .246 \\
\hline Diabetes & $3(12.5 \%)$ & $10(13.3 \%)$ & 13 & .916 \\
\hline PVD* & $7(29.2 \%)$ & $9(12.0 \%)$ & 16 & .047 \\
\hline Renal insufficiency & $4(16.7 \%)$ & $16(21.3 \%)$ & 20 & .62 \\
\hline Prior cardiac surgery & $3(12.5 \%)$ & $16(21.3 \%)$ & 19 & .339 \\
\hline Previous aortic surgery & $7(29.2 \%)$ & $9(12.0 \%)$ & 16 & .047 \\
\hline
\end{tabular}

$C A D$, coronary artery disease; $C O P D$, chronic obstructive pulmonary disease; HTN, hypertension; $P V D$, peripheral vascular disease. *PVD as defined by carotid artery stenosis, abdominal aortic aneurysm, claudication, and history of leg ischemia requiring intervention.

Table 1 . There was a significantly higher incidence of chronic obstructive pulmonary disease, peripheral vascular disease, and previous aortic surgery in the early-generation cohort.

\section{Aneurysm Profile}

Details of the aneurysm profile are depicted in Table 2. There was no significant difference in the mean size of aneurysm, percentage of symptomatic aneurysms, and percentage of saccular aneurysm in the early-generation versus late-generation cohorts. Aneurysms were almost all exclusively atherosclerotic in cause. Aortic dissections, mycotic, and traumatic aneurysms were not included in these trials. Aneurysm location was classified as type A (left subclavian to T6), 30\%; type B (midthoracic aorta to celiac axis), 31\%; and type C (left subclavian to celiac axis), 35\% (Figure 1). There was no significant difference in aneurysm location between the early and late-generation cohorts $(P>.2)$.

\section{Early Outcome}

Perioperative mortality. Operative mortality, defined as death within 30 days of the procedure or during the same hospital admission, was $5.1 \%(\mathrm{n}=5)$ for the entire study population. There was no difference in early-generation $(4.2 \%)$ versus late-generation $(5.3 \%)$ cohorts $(P=.9)$. The causes of death were respiratory failure (1), myocardial infarction (1), sudden cardiac arrest (1), sepsis (1), and postoperative paraplegia and renal failure (1) (this patient ultimately died of multisystem organ failure).

Procedural data. All 99 patients had successful deployment of the device, and no patients required conversion to open repair. The mean number of devices used per patient was 2.62 (3.04 vs 2.49 , early vs late generation, $P=.07$ ). The left subclavian was covered in 20 cases $(20.8 \%$ vs $21.3 \%$, early vs late generation, $P=.96$ ) after a scheduled elective carotid subclavian transposition or bypass. The site of vascular access was the femoral artery in 68 cases, iliac artery in 28 cases, and infrarenal aorta in 2 cases (early vs late generation, $P>.15$ ). Nineteen patients had a conduit constructed to insert the device $(20.8 \%$ vs $18.7 \%$ early generation vs late generation, $P=.82$ ).

Overall, $11 \%$ of patients required mechanical ventilation more than 24 hours $(20.8 \%$ vs $8 \%$, early vs late generation, $P=.08)$. The incidence of renal failure was $3 \%(8.3 \%$ and $1.3 \%$, early vs late generation, $P=.08$ ).

TABLE 2. Aneurysm profile

\begin{tabular}{|c|c|c|c|c|}
\hline & $\begin{array}{l}\text { Early generation } \\
\qquad \mathbf{N}=24\end{array}$ & $\begin{array}{l}\text { Late generation } \\
\qquad N=75\end{array}$ & $\begin{array}{c}\text { Overall University of } \\
\text { Pennsylvania experience } \\
\mathrm{N}=99\end{array}$ & $\begin{array}{c}P \text { value } \\
\text { (early vs late generation) }\end{array}$ \\
\hline Symptomatic & $12(50 \%)$ & $30(40 \%)$ & 42 & .388 \\
\hline Saccular aneurysm & $7(29.2 \%)$ & $28(37.3 \%)$ & 35 & .466 \\
\hline \multicolumn{5}{|l|}{ Aneurysm size } \\
\hline Mean & 65.2 & 63.2 & 63.68 & .472 \\
\hline Range & $45-99$ & $30-105$ & $30-105$ & \\
\hline
\end{tabular}




\section{Neurologic Complications}

Spinal cord ischemia. The total incidence of spinal cord ischemia was $5.1 \%$, with no significant difference between early-generation (4.2\%) and late-generation $(5.3 \%)$ cohorts. There was 1 case of paraplegia, 3 cases of paraparesis, and 1 case of only intraoperative loss of somatosensory evoked potentials while under general anesthesia, which was reversed with blood pressure management and cerebrospinal fluid drainage. Three of the 5 patients had a full recovery, for an incidence of permanent spinal deficit of $2 \%$. Four of 5 patients with spinal ischemia had type $\mathrm{C}$ coverage, and 1 patient had a previous abdominal aortic aneurysm repair.

Stroke. One stroke occurred in the early-generation cohort, and 4 strokes occurred in the late-generation cohort $(P=.675)$, for an overall incidence of $5 \%$. All 5 patients had type A or C aortic coverage, and 3 of the 5 patients had a preoperative history of cardiovascular accident. None of the 5 patients had the left subclavian artery covered. Two of the 5 patients made a complete recovery with no deficit at discharge.

Vascular access complications. Vascular complications occurred in $41.7 \%(\mathrm{n}=10)$ of the early-generation cohort and $22.7 \%(\mathrm{n}=17)$ of the late-generation cohort $(P=$ $.069)$, for an overall incidence of $27 \%(\mathrm{n}=27)$. Of these 27 cases, repair involved an interposition graft in $41 \%$, a patch angioplasty in 33\%, and a bare metal stent repair in $19 \%$.

\section{Late Outcome}

Through the 6-year period of this study, radiologic follow-up was complete in $86 \%$ of patients and vital statistics data were available in $100 \%$.

Overall survival. For the entire cohort, 24 of the 99 patients died during the 6-year follow-up. Kaplan-Meier analysis estimated that 1,3 , and 5-year survival was $84.5 \%$, $70.5 \%$, and $52.4 \%$, respectively. Five-year survival was $45 \%$ for the early-generation cohort and 55\% for the late-generation cohort $(P=.24)$ Figure 2.

Freedom from aneurysm-related event. The KaplanMeier estimate of freedom from aneurysm-related events, as defined by freedom from endoleak, aortic rupture, aortic dissection, or reintervention on the aorta, was $73 \%, 69 \%$, and $64 \%$ at 1,3 , and 5 years, respectively.

Three patients had an aneurysm-related death after the perioperative period:

- Patient 1 had an early-generation endograft. He had an endoleak that was never successfully covered despite reintervention. The patient's aorta was tortuous with 90-degree angles that could not be navigated by the early-generation device. He had an aortic rupture while he was still in the hospital after an endovascular reintervention.

- Patient 2 had an early-generation endograft and a tortuous aorta. He had a persistent endoleak and pre-

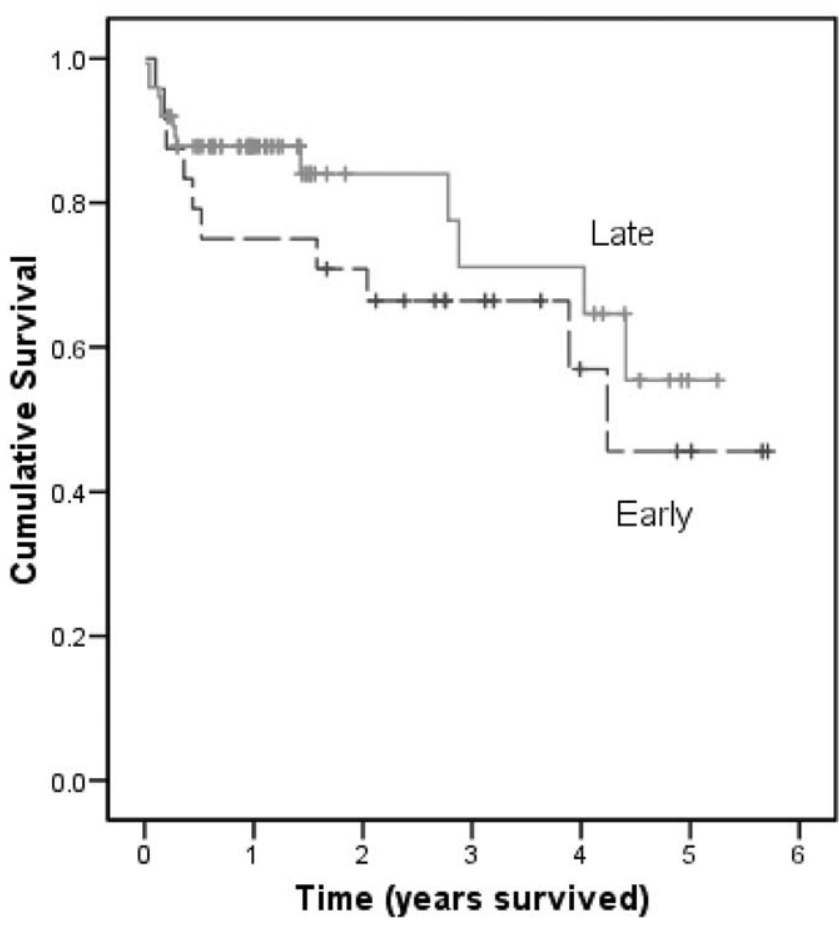

Figure 2. Kaplan-Meier 5-year survival, early versus late generation.

sented 2 years postoperatively with graft migration and subsequently a kinked graft. He was offered open surgery but refused; he subsequently died of aortic rupture.

- Patient 3 had a late-generation endograft. He presented to the hospital approximately 1 month postoperatively with a type A dissection that started at the proximal endograft landing zone and extended in a retrograde fashion to the ascending aorta. He underwent urgent open surgical repair of the type A dissection. At surgery, it was discovered that the bare spring flares of the endograft had perforated the aortic wall. The dissection was repaired with a total arch replacement using a 4-branch graft. The distal part of the branch graft was anastomosed to the endograft. This patient died postoperatively of multisystem organ failure.

\section{Endoleaks}

Early. At 30-day follow-up, 23 endoleaks were detected in 22 patients $(45.8 \%$ and $14.7 \%$, early vs late generation, $P=.001)$. Type I endoleaks accounted for $57 \%(\mathrm{n}=13)$, type II endoleaks accounted for $26 \%(\mathrm{n}=6)$, and type III endoleaks accounted for $17 \%(\mathrm{n}=4)$. There were no type IV endoleaks observed. Of 22 patients with an endoleak, 7 had reinterventions, 4 had an endoleak resolve spontaneously (3 within 6 months; resolution took 2 years in 1 
patient receiving Coumadin), 3 were lost to follow-up, and 8 have endoleaks that are currently ongoing and being followed.

Late. A late endoleak developed in 3 patients (3\%) that was not present on the 30-day follow-up CT. One patient underwent successful urgent surgical intervention 3 years after endograft placement for a symptomatic (contained rupture) endoleak at the proximal landing zone. One patient ( 1 year after endograft insertion) is awaiting surgical correction at the time of this writing. One patient (1 year after endograft insertion) is currently undergoing workup.

Reinterventions. Ten patients $(10.1 \%)$ underwent reintervention on the aorta (25\% and $5.3 \%$, early vs late generation, $P=.012$ ). Of these 10 patients, 7 had a reintervention for endoleak soon after their 30-day CT scan. Two patients required surgical reintervention on their aorta (discussed previously, 1 patient for late endoleak and 1 patient for dissection caused by the stent graft). One patient had endovascular reintervention for a new aneurysm that was not present at the initial procedure.

Of the 7 patients who underwent reintervention for an endoleak on their 30-day CT, all had endovascular procedures, 6 with graft extensions and 1 with a coil embolization of a type II endoleak. Four of the 7 patients had persistent leaks on follow-up CT despite the reintervention.

\section{Discussion}

The feasibility of endovascular repair of DTA using covered stents was reported in $1994 .^{3}$ Since then, thoracic aortic endografts have been used with early success in small to moderate-sized retrospective single-center series. ${ }^{4-9}$ Six years ago we enrolled our first patient in an FDA-approved thoracic stent graft trial.

During the 6-year period, 99 patients received 5 different endograft models made by 2 manufacturers. For the purpose of this article, 2 of these models were classified as earlygeneration devices (24 patients) and 3 of these models were classified as late-generation devices (75 patients) on the basis of the mechanics of the device.

The patients in the early-generation cohort were part of a Phase I study and thus not candidates for open surgical repair. As expected, they had a significantly higher risk profile as manifested by an increased incidence of chronic obstructive pulmonary disease, peripheral vascular disease, and previous aortic surgery. However, aneurysm morphology, as defined by location, size, percentage who are symptomatic, and percentage who require preoperative carotid subclavian bypass/transposition, was similar between the 2 cohorts.

Our series and the series in the literature show low perioperative mortality. Although minimally invasive, endografting of DTA is not without negative perioperative outcomes.

\section{Specific Findings of This Study}

The main differences in outcomes between the earlygeneration and late-generation cohorts were noticed in the incidence of endoleaks, reinterventions, and vascular complications. In this study, results over time and with newer generation devices did not improve in regard to perioperative mortality, stroke, or spinal ischemia.

Endoleaks post-DTA stent grafting are a poorly understand phenomenon. In this series, $22 \%$ of patients were documented to have an endoleak by a radiologist at their first follow-up CT. The incidence of endoleaks was markedly higher in the early-generation versus the lategeneration cohort $(45.8 \%$ vs $14.7 \%$, respectively, $P=$ .001). Both cohorts had similar incidence of subclavian transposition, distribution of access site vessels, and aneurysm size, indicating that the aortic morphology was comparable in the cohorts. The lower incidence of endoleaks in the late-generation cohort likely reflects that the later generation of devices are easier to deploy at the curved arch and tortuous distal aorta, allowing more precise proximal landing zone deployment. Another reason for improved results with endoleaks is that with more experience we are covering more aorta, being more aggressive with overlapping, and using tapered grafts more judiciously. In addition, as our experience has progressed we have been more aggressive in fixing endoleaks intraoperatively and not leaving the operating theatre if there is an endoleak. This is similar to open surgery, in which we now know that after valvular repair/ replacement, paravalvular leaks do not improve and lead to worse long-term outcome.

Vascular complications are sometimes underestimated with this minimally invasive technology. The overall incidence in this series was $27 \%$ (41.7\% and $22.7 \%$ in early and late-generation cohorts, respectively). There was a strong trend, although this value did not reach statistical significance $(P=.069)$. The early-generation devices all required multiple exchanges, which can lead to traumatic peripheral vascular effects. The endograft devices are inserted through sheaths that measure $20 \mathrm{~F}$ to $25 \mathrm{~F}$ in diameter and can cause access problems in certain patients. When the femorals are of small diameter or heavily calcified, we find it prudent to access the iliac vessels through a retroperitoneal approach rather than cannulate the femorals. In addition, consideration should be given to sewing on a conduit prophylactically for marginal access anatomy. Significant ileofemoral and infrarenal aortic complications can occur with thoracic endografts. In this study, ileofemoral bypass procedures, angioplasty, and bare stenting were all reported as vascular complications. Thus, it is important to have team expertise in this area.

Spinal cord ischemia is the most dreaded and feared complication of DTA repair. Early reports indicated an incidence of up to $30 \%$ with open surgical repair. More 
recent published data from 2 expert high-volume centers that specialize in open surgical repair of DTA report mortality rates in the range of $4 \%$ to $9 \%$ and paraplegia rates of less than $3 \%$ for isolated DTAs. ${ }^{10,11}$ Despite these publications it is hard to judge what the true incidence of spinal ischemia is today with open repair of DTA. Most centers end up doing small volumes, surgical techniques are variable, there is no central registry, and undesirable outcomes are frequently underpublished. In the first completed multicenter comparative trial of endovascular versus an open surgical repair of DTA, the incidence of spinal ischemia was $2.9 \%$ in the endograft cohort and $13.8 \%$ in the open surgical control cohort. ${ }^{12}$

When endografting of DTA became a technically feasible option, we hypothesized that 1 of the proposed benefits, in addition to the minimally invasive nature, may be a decreased perioperative incidence of spinal ischemia. Theoretic reasons for lower spinal cord ischemia rates with an endovascular technique include the following:

- no period of aortic crossclamping and resultant nonpulsatile perfusion

- fewer periods of perioperative hypotension associated with blood loss or hemodynamic shifts

- ability to tolerate higher mean arterial pressures because there are no suture lines

- earlier awakening from general anesthesia, which allows one to tailor blood pressure management to neurologic examination

Conversely, one could postulate that an open technique may confer an advantage for the following reasons.

- Intercostals, which would be sacrificed with stent grafting, can be reattached or intercostals that are not reattached would at the very least be oversewn and not cause spinal steal.

- The extent of aorta replaced may be greater with stent grafting because the seal on the stent graft is better when the landing zone is further away from the true aneurysm. ${ }^{13}$

- The large sheath insertion required for endografting increases the chance of injury to iliac vessels, which supply important collaterals to the spinal cord from the hypogastric and pelvic vascular plexus.

The incidence of spinal ischemia in this series was 5.1\%, with no difference between the early and late-generation cohorts. This is comparable to the incidence of $3.6 \%$ to $6.5 \%$ in the published literature. ${ }^{6,14-16}$ Three of the 5 patients fully recovered by following a published paradigm for management of spinal ischemia. ${ }^{17-19}$ Thus, the incidence of permanent spinal cord ischemia after endografting in this series was $2 \%$.

The incidence of stroke was 5\%. In a multicenter comparative trial of endografting versus open surgical repair, we previously reported a higher incidence of stroke in patients with subclavian-carotid transposition or bypass and postulated that it may be an indication of higher atherosclerotic burden at the level of the arch. However, in this study none of the 5 patients with stroke underwent a subclaviancarotid transposition or bypass preoperatively. Thus, the incidence and cause of stroke with endografting require further clarification.

Freedom from an aneurysm-related event, as defined by freedom from any endoleak, aortic rupture, dissection, or aortic reintervention, was $64 \%$ at 5 years. There were 3 cases of frank aortic rupture; 2 were related to endoleaks, and 1 was caused by an uncovered nitinol stent that ruptured the aorta proximally and led to a retrograde dissection. Retrograde type A dissection from thoracic aortic stent grafting has been reported in the literature. ${ }^{20}$ We now generally try to avoid the use of uncovered stents.

The Kaplan-Meier estimated 1, 3, and 5-year survival was $84.5 \%, 70.5 \%$, and $52.3 \%$, respectively, which is consistent with results of thoracic endografting reported in the literature. ${ }^{6,12,20}$ Aneurysm-related mortality after the perioperative period was $3 \%$. This indicates that patients with thoracic aortic aneurysms have complex diseases and die of comorbidities unrelated to their thoracic aortic aneurysm. These patients require close follow-up in a multidisciplinary fashion. In a thought-provoking article, Demers and colleagues ${ }^{21}$ at Stanford questioned whether asymptomatic patients who are deemed to be inoperable for open repair should have even been treated with endografting because survival in this group was bleak. In this study, when patients with symptomatic aneurysms were compared with patients with asymptomatic aneurysms (some of whom were deemed "inoperable") there was no difference in survival. This topic warrants further investigation.

\section{Conclusions/Lessons Learned}

- Vascular complications do occur, but their incidence is decreasing with new devices. Team expertise is required to deal with them.

- The incidence of endoleaks and reinterventions is decreasing with newer generation devices. Endoleaks usually occur early, but as experience progresses, late endoleaks are being seen.

- Long-term outcome of endografts in the thoracic aorta is unknown. There is an incidence of complications and reintervention. These grafts should be used with caution in young patients. All patients who receive a thoracic endograft require mandatory lifelong radiologic follow-up.

- As thoracic endografts receive FDA approval and become readily available, caution needs to be exercised. We agree wholeheartedly with Dr Verdant's comments $^{22}$ when he mentioned that these operations should be conducted in "... highly specialized centres 
for aortic surgery equipped with fully trained personnel and optimal methods of organ protection."

- On the basis of these data, we can expect to see improved results as device designs improve. These data support a challenge to the industry to continue development of thoracic aortic disease-specific stent designs.

\section{References}

1. Hamerlijnck RP, Rutsaert RR, De Geest R, Brutel de la Riviere A, Defauw JJ, Vermeulen FE. Surgical correction of descending thoracic aortic aneurysms under simple aortic cross-clamping. J Vasc Surg. 1989;9:568-73.

2. Huynh TTT, Miller CC, Estrera AE, Porat EE, Safi HJ. Thoracoabdominal and descending thoracic aortic aneurysm surgery in patients aged 79 years or older. J Vasc Surg. 2002;36:469-75.

3. Dake MD, Miller DC, Semba CP, et al. Transluminal placement of endovascular stent-grafts for the treatment of descending thoracic aortic aneurysms. N Engl J Med. 1994;331:1729-34.

4. Ramaiah V, Rodriguez-Lopez J, Diethrich EB. Endografting of the thoracic aorta: single center experience with technical considerations. $J$ Card Surg. 2003;18:444-54.

5. Ehrlich M, Grabenwoeger M, Cartes-Zumelzu F, et al. Endovascular stent graft repair for aneurysms on the descending thoracic aorta. Ann Thorac Surg. 1998;66:19-25.

6. Ellozy SH, Carroccio A, Minor M, et al. Challenges of endovascular tube graft repair of thoracic aortic aneurysm: midterm follow up and lessons learned. J Vasc Surg. 2003;38:676-83.

7. Lepore V, Lonn L, Delle M, et al. Treatment of descending thoracic aneurysms by endovascular stent grafting. J Card Surg. 2003;18: 436-43.

8. Inoue $\mathrm{K}$, Iwase $\mathrm{T}$, Sato $\mathrm{M}$, et al. Clinical application of transluminal endovascular graft placement for aortic aneurysms. Ann Thorac Surg. 1997;63:522-8.

9. Criado FJ, Clark NS, Barnatan MF. Stent graft repair in the aortic arch and descending thoracic aorta: a 4-year experience. J Vasc Surg. 2002;36:1121-8.

10. Coselli JS, LeMaire SA, Conklin LD, Adams GJ. Left heart bypass during descending thoracic aortic aneurysm repair does not reduce the incidence of paraplegia. Ann Thorac Surg. 2004;77:1298-303.

11. Estrera AL, Rubenstein FS, Miller CC, et al. Descending thoracic aortic aneurysm: surgical approach and treatment using the adjuncts cerebrovascular fluid drainage and distal aortic perfusion. Ann Thorac Surg. 2001;72:481-6.

12. Bavaria JE, Appoo JJ, Makaroun MS, et al. Endovascular stent grafting versus open surgical repair of descending thoracic aortic aneurysms: a multi-center comparative trial. J Thorac Cardiovasc Surg. Submitted.

13. Bavaria JE. Invited commentary on: descending thoracic aortic aneurysm: surgical approach and treatment using the adjuncts cerebrospinal fluid drainage and distal aortic perfusion. Ann Thorac Surg. 2001;72: 486.

14. Gravereaux EC, Faries PL, Burks JA, et al. Risk of spinal cord ischemia after endograft repair of thoracic aortic aneurysms. $J$ Vasc Surg. 2001;34:997-1003.

15. Mitchell RS, Miller DC, Dake MD. Stent graft repair of thoracic aortic aneurysms. Semin Vasc Surg. 1997;10:257-71.

16. Leurs LJ, Bell R, Degrieck Y, et al. Endovascular treatment of thoracic aortic diseases: combined experience from the EUROSTAR and United Kingdom Thoracic Endograft registries. J Vasc Surg. 2004;40: 670-80.

17. Cheung AT, Pochettino A, Guvakov DV, et al. Safety of lumbar drains in thoracic aortic operations performed with extracorporeal circulation. Ann Thorac Surg. 2003;76:1190-7.

18. Cheung AT, Weiss SJ, McGarvey ML, et al. Interventions for reversing delayed-onset postoperative paraplegia after thoracic aortic reconstruction. Ann Thorac Surg. 2002;74:413-21.
19. Cheung AT, Pochettino A, McGarvery ML, et al. Strategies to manage paraplegia risk after endovascular stent repair of descending thoracic aortic aneurysms. Ann Thorac Surg. 2005;80:1280-9.

20. Bortone AS, De Cillis E, D'Agostino D, Schinosa LLT. Endovascular treatment of thoracic aortic disease. Four years of experience. Circulation. 2004;110(suppl II):II-262-II-7.

21. Demers P, Miller DC, Mitchell RS, et al. Midterm results of endovascular repair of descending thoracic aortic aneurysms with first generation stent grafts. J Thorac Cardiovasc Surg. 2004;127:664-73.

22. Verdant A. Regarding "Challenges of endovascular tube graft repair of thoracic aortic aneurysm: midterm follow up and lessons learned." J Vasc Surg. 2004:39;1145.

\section{Discussion}

Dr. Karmy-Jones. In the interest of disclosure I need to point out the fact that a fellow Albertan has presented this paper in no documentable way has biased my review of the paper.

The authors have presented as you have heard their six-year experience with endograft management, specifically with atherosclerotic thoracic aneurysms. It is significant in my mind that their group has in cooperation with their vascular colleagues developed a multidisciplinary treatment program that is incorporated into their training program for future cardiothoracic residents.

Endografts are associated with their own specific technical complications, notably vascular access complications, stroke and endoleak. The gist of this paper is that so-called newer generation grafts, because of more flexible delivery systems and graft material, are associated specifically with decreased complications in vascular access sites, endoleak and improvement in late mortality. Because this is a technique-specific paper, my questions that start off the discussion, five of them, are specifically technical.

The first is, are the strokes related to their need to cover the left subclavian and/or are they related to wire manipulation in the proximal descending thoracic aorta?

Dr. Appoo. That's a very interesting question. All patients where the left subclavian was covered had a preoperative either carotid subclavian bypass or subclavian transposition. In previous work, in a multicenter looking at comparisons between open and endovascular repair, we had shown that 4 out of 5 patients in the endovascular group all had preoperative subclavian transposition indicating what we thought was a higher atherosclerotic burden up at the distal arch and strokes are associated with that. But in this study, none of the five patients who had a stroke actually had preoperative subclavian transposition.

Dr. Karmy-Jones. So it's a little unclear.

Dr. Appoo. It's unclear. I mean we presume that was an indication of higher atherosclerotic burden in those patients having that procedure but that wasn't the case in this situation. As to the wire management, I'm sure that's part of it. We are putting wires all the way up the arch and into the ascending aorta at times in these patients.

Dr. Karmy-Jones. Preoperatively, it leads into the next question, is that, it is an important issue for developing endovascular programs, but how do you assess cerebral circulation preoperatively. Do you favor cerebral angiography or TCD?

Dr. Appoo. We use cerebral angiography to ensure patency of both vertebral arteries.

Dr. Karmy-Jones. I accept your argument that the newer generation grafts are more flexible, etc., but is this benefit across the board 
with the graft, Gore versus Medtronic, or do you have differences that will shape the choice in any given case? Or can you tell?

Dr. Appoo. We tried very hard in this study not to compare one manufacturer versus another. I think these newer devices have both. They have improvements across and then they have specific things. I think with the Gore device the major advantage is that it is a single exchange device where you don't have to manipulate the vascular access site again and again. The Medtronic has advantages in that it does provide a larger graft, so if you have a patient with a large aorta, even where it is supposedly normal caliber, sometimes that is too big for the Gore device. There are specific benefits of each.

Dr. Karmy-Jones. Did the use of a conduit increase, decrease or have no effect on the incidence of vascular access complications?

Dr. Appoo. Our overall use of conduits was $19 \%$, similar in both groups. Since it was similar in both groups, we can't assume that it improved it but I think maybe our employment of conduits was more judicious.
Dr. Karmy-Jones. Finally, you have noted a greater incidence of comorbidities, particularly COPD, prior aortic surgery, peripheral vascular disease in the earlier group. How is it possible to discriminate between potential selection bias and the benefit of the newer generations given that comparison?

Dr. Appoo. Inclusion/exclusion criteria were different for each of the three FDA studies. As we talked about, phase I study was high risk. The others were high and low risk. I think that is part of it in terms of outcome but the comorbidities probably play a larger role in outcome measurements such as stroke, operative mortality, and long-term outcome. I think device-specific outcomes, things like vascular complications and endoleaks, are probably irrespective of the comorbidities in the different patient populations.

Dr. Karmy-Jones. I'd like to thank the association for the privilege and the pleasure of reviewing this paper. Thank you. 